\section{CPS-294 EFFICACY AND SAFETY PROFILE OF TRIFLURIDINE- TIPIRACIL AND REGORAFENIB IN THE TREATMENT OF METASTATIC COLORECTAL CANCER}

L Cantarelli*, JA Morales Barrios, F Gutierrez Nicolas, S Garcia Gil, B Del Rosario Garcia, GJ Nazco Casariego. Complejo Hospitalario Universitario De Canarias, Pharmacy, Santa Cruz De Tenerife, Spain

\subsection{6/ejhpharm-2021-eahpconf.126}

Background and importance Regorafenib (REG) and trifluridine-tipiracil (TAS-102) are used in metastatic colorectal cancer (mCRC) after failure of conventional therapy based on fluorouracil (5-FU) schemes.

Aim and objectives To evaluate the efficacy and safety of TAS102 and regorafenib drugs in patients with mCRC.

Material and methods A retrospective single centre study was conducted from January 2010 to August 2020, which included all patients diagnosed with CRBM treated with TAS-102/REG. Clinical and demographic variables were collected, corresponding to age, sex, time of disease follow-up, time of treatment with the drug, previous adjuvant/neoadjuvant, presence of RAS type mutation and number of previous metastatic lines.

Efficacy was determined by calculating progression free survival (PFS) applying the Kaplan-Meyer statistic with SPSS V.15. Progression was analysed according to the radiological criteria response evaluation criteria in solid tumours (RECIST V.1.1). The occurrence of grade III/IV adverse effects (AEs) leading to early dose reduction/suspension of treatment was determined. AEs were classified according to the common terminology criteria for adverse effects (CTCAE V.6.0).

Results 104 patients were included, 57.7\% $(\mathrm{n}=60)$ treated with REG $(66.3 \%, \mathrm{n}=69)$; mean age was 63.9 years $(41-83))$. Mean follow-up time of the disease was 3.9 years (0.1-15.5). Mean duration of treatment was 3.9 months for TAS-102 and 4.2 for REG. $46 \%(n=48)$ of patients received adjuvant therapy and $16.3 \% \quad(n=17)$ neoadjuvant. $48.1 \%$ presented RAS mutation $(\mathrm{n}=50)$. Mean of previous metastatic lines was 2.4 (1-7).

$84 \%(\mathrm{n}=37)$ of patients progressed with TAS-102 versus $72 \%(n=43)$ with REG. $11.5 \%(n=12)$ discontinued treatment due to toxicity. Median PFS was the same for both: 3.8 months $(p=0.86)$. A reduction in drug doses due to the appearance of AEs was carried out in $25 \%$ of cases $(n=11)$ with TAS-102 versus $61.7 \%$ with REG $(n=37)$. The most common grade III/IV AEs with TAS-102 were haematological toxicity $(20.5 \%, \mathrm{n}=9)$, gastrointestinal $(2.3 \%, \mathrm{n}=1)$ and other $(2.3 \%, \mathrm{n}=1)$; for REG, gastrointestinal $(16.7 \%, \mathrm{n}=10)$, asthenia $(13.3 \%, \mathrm{n}=8)$, skin toxicity $(11.7 \%, \mathrm{n}=7)$, mucositis $(6.7 \%, n=4)$, plaquetopenia $(3.3 \%, n=2)$ and other $(13.3 \%$, $\mathrm{n}=8)$.

Conclusion and relevance The study showed that there were no significant differences between PFS values between TAS102 and REG. However, treatment with REG was tolerated worse, with AEs in more than $60 \%$ of cases compared with $25 \%$ with TAS- 102 .

\section{REFERENCES AND/OR ACKNOWLEDGEMENTS}

Conflict of interest No conflict of interest

\section{CPS-295 IMMUNOTHERAPY FOR CANCER THERAPEUTIC POSITIONING REPORTS: KNOWLEDGE, EXPECTATIONS AND EXPERIENCES THROUGH A NATIONAL SURVEY}

${ }^{1} \mathrm{M}$ Lafuente Gonzalez*, ${ }^{2} \mathrm{JF}$ Marin Pozo, ${ }^{3} \mathrm{E}$ Gonzalez-Haba Peña, ${ }^{4} \mathrm{AC}$ Cercos Lleti. ${ }^{1}$ Spanish Agency of Medicines and Medical Devices AEMPS, Pharmacology and Clinical Assessment Division. Medicines for Human Use Department, Madrid, Spain; ${ }^{2}$ Complejo Hospitalario De Jaen, Pharmacy Service, Jaen, Spain; ${ }^{3}$ Hospital Gregorio Marañón, Pharmacy Service, Madrid, Spain; ${ }^{4}$ Universitary Hospital Dr Peset, Pharmacy Service, Valencia, Spain

\subsection{6/ejhpharm-2021-eahpconf.127}

Background and importance In our country, considerable progress has been made in recent years since the establishment of the clinical therapeutic position reports (TPR) in 2013, but more research is needed about their impact on decision making.

Aim and objectives To determine the value of immunotherapy for cancer therapeutic positioning reports (ITc-TPR) in clinical practice.

Material and methods A nationwide survey was designed for hospital pharmacists and clinical oncologists of the ITcTPR, published between May 2013 and March 2020. The main variables collected were: sociodemographics of the hospitals and health professionals, approval criteria and variables related to the acceptance of the ITc-TPR recommendations.

Results During the study period, 46 ITc-TPR of 22 active substances were published. 27 health professionals answered the survey, $81.5 \%$ hospital pharmacists and $18.5 \%$ oncologists, ascribed to 24 hospitals in the national territory. $33.3 \%$ of the professionals had participated in the development of some TPR-ITc. In $45.8 \%$ of hospitals, incorporation of ITc was decided at the regional level, and in $66.7 \%$ of hospitals the drug and therapeutic indication with ITc-TPR published should be re-evaluated by the pharmacy and therapeutics hospital commission. In 50\%, the authorised indications were the same as those of the ITc-TPR and $70.8 \%$ followed the recommendations of the ITc-TPR.

$48.1 \%$ of the professionals who responded to the survey believed that ITc-TPR have eliminated barriers for access of patients to ITc, have decreased the variability of clinical care and promoted equity in the national territory. $66.7 \%$ believed the incorporation of the drug into clinical practice was faster if there was a published ITc-TPR. The main limitations for patients to access ITc were: not financed by the national health system $(55.6 \%)$, high cost $(37.1 \%)$ and the restrictions at the autonomic level (29.6\%). The aspects of the ITc-TPR that should be improved were: pharmacoeconomic evaluation $(66.7 \%)$, conclusions useful for clinical practice $(70.4 \%)$, a single evaluation at the national level $(70.4 \%)$ and decisions binding (80.5\%).

Conclusion and relevance ITc-TPR is a useful and well established tool at the national level for the positioning of a new drug. The results of the survey will allow the development of strategies to improve ITc-TPR.

\section{REFERENCES AND/OR ACKNOWLEDGEMENTS}

Acknowledgements: AEMPS, GEDEFO, participants, directors, teachers and tutors of Máster Inmunoterapia del Cáncer. Conflict of interest No conflict of interest 